OPEN ACCESS

Edited by:

Christophe Le May,

Agrocampus Ouest, France

Reviewed by:

Sabine Banniza,

University of Saskatchewan, Canada

Fournet Sylvain,

INRA (Paris), France

*Correspondence:

Fabien Halkett

fabien.halkett@inra.fr

${ }^{\dagger}$ Present address:

Michaël Pernaci,

PalmElit SAS, Montferriez-sur-Lez,

France

Anne-Laure Boutigny,

ANSES - Laboratoire de la Santé des Végétaux, Unité de Bactériologie,

Virologie et OGM, Angers, France

Specialty section: This article was submitted to

Plant Microbe Interactions,

a section of the journal

Frontiers in Plant Science

Received: 20 April 2018

Accepted: 03 September 2018

Published: 28 September 2018

Citation:

Maupetit A, Larbat R, Pernaci $M$ Andrieux A, Guinet $C$, Boutigny $A-L$, Fabre B, Frey $P$ and Halkett $F$ (2018)

Defense Compounds Rather Than

Nutrient Availability Shape

Aggressiveness Trait Variation Along

a Leaf Maturity Gradient in a

Biotrophic Plant Pathogen.

Front. Plant Sci. 9:1396.

doi: 10.3389/fpls.2018.01396

\section{Defense Compounds Rather Than Nutrient Availability Shape Aggressiveness Trait Variation Along a Leaf Maturity Gradient in a Biotrophic Plant Pathogen}

\author{
Agathe Maupetit ${ }^{1}$, Romain Larbat ${ }^{2}$, Michaël Pernaci ${ }^{1+}$, Axelle Andrieux ${ }^{1}$, Cécile Guinet ${ }^{3}$, \\ Anne-Laure Boutigny ${ }^{3+}$, Bénédicte Fabre ${ }^{1}$, Pascal Frey ${ }^{1}$ and Fabien Halkett ${ }^{1 *}$
}

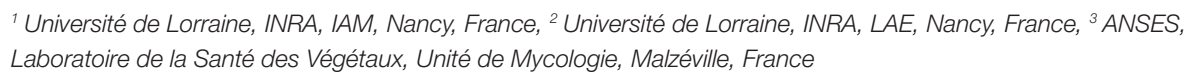

Foliar pathogens face heterogeneous environments depending on the maturity of leaves they interact with. In particular, nutrient availability as well as defense levels may vary significantly, with opposing effects on the success of infection. The present study tested which of these factors have a dominant effect on the pathogen's development. Poplar leaf disks of eight maturity levels were inoculated with the poplar rust fungus Melampsora larici-populina using an innovative single-spore inoculation procedure. A set of quantitative fungal traits (infection efficiency, latent period, uredinia size, mycelium quantity, sporulation rate, sporulation capacity, and spore volume) was measured on each infected leaf disk. Uninfected parts of the leaves were analyzed for their nutrient (sugars, total $\mathrm{C}$ and $\mathrm{N}$ ) and defense compounds (phenolics) content. We found that M. larici-populina is more aggressive on more mature leaves as indicated by wider uredinia and a higher sporulation rate. Other traits varied independently from each other without a consistent pattern. None of the pathogen traits correlated with leaf sugar, total $\mathrm{C}$, or total $\mathrm{N}$ content. In contrast, phenolic contents (flavonols, hydroxycinnamic acid esters, and salicinoids) were negatively correlated with uredinia size and sporulation rate. The pathogen's fitness appeared to be more constrained by the constitutive plant defense level than limited by nutrient availability, as evident in the decrease in sporulation.

Keywords: constitutive defense, aggressiveness traits, plasticity, nutrient availability, rust fungus

\section{INTRODUCTION}

Plant diseases and pests are responsible for crop damage that may account for up to $40 \%$ of yield losses worldwide (Boonekamp, 2012). Pathogen aggressiveness, the quantitative component of pathogenicity (Pariaud et al., 2009a), has been widely studied in order to forecast the evolutionary potential of plant pathogens and to better design disease control strategies (Lannou, 2012). Aggressiveness can be evaluated at different scales: by measuring the epidemic rate at the field scale or through elementary phenotypic traits at the individual scale (Pariaud et al., 2009a). The environment plays a key role in developmental processes of the pathogen and influences the individual's phenotype and the scale of fitness (Scheiner, 1993; Jarosz and Davelos, 1995). 
Biotrophic fungal pathogens, such as rust and powdery mildew fungi, spend most of their life cycle within living plant tissues, so their fitness is directly affected by leaf physiology (Agrios, 2005; Gamm et al., 2011; Duplessis et al., 2013). On the one hand the leaf tissue provides the pathogen with the necessary nutrient resources for its development. On the other hand, it impedes pathogen development through a battery of plant defense compounds (Ullah et al., 2017).

Studies on interactions between plants and their foliar pathogens have shown that plant age, and especially leaf age, leads to different responses of the pathogen. Coleman (1986) postulated from a series of experiments that leaves pass through a peak of susceptibility to disease and pest attack. He proposed that for many stresses, including infection by biotrophic fungi, this peak coincides with changes in leaf properties during the transition from metabolite sink to source. This stage corresponds to a maximal nutrient availability. After this sinksource transition, free sugar content and thus nutrient resources decrease with increasing leaf maturity (physiological stage at a relative leaf age). Many studies support this view, demonstrating a maximum disease level before leaves are fully expanded and a decrease of disease severity on older leaves, which is also referred to as ontogenetic resistance (Ficke et al., 2002; Xavier et al., 2015; Farber and Mundt, 2017). In agreement, Merry et al. (2013) demonstrated that the peak in the severity of powdery mildew along a primary shoot of Vitis vinifera corresponds to the position of the first leaf that does not import carbohydrates. However, some studies have reported a contrasting pattern, e.g., Knott and Mundt (1991) found that infection efficiency of the wheat leaf rust fungus Puccinia recondita f. sp. tritici is $25 \%$ higher on older than on younger leaves in five wheat cultivars. The opposite trend may involve the dark side of plant-pathogen interaction: defense compounds. Their actions on a pathogen's development have been studied in several pathosystems, including rust fungi infecting poplar (Johnson and Kim, 2005), black currant (Vagiri et al., 2017), and willow (Hakulinen et al., 1999). Phenolic compounds present in leaves appear to highly influence the general plant resistance to fungi (Adandonon et al., 2017; Ullah et al., 2017). Most studies have focused on plant susceptibility to the disease (i.e., infection efficiency of the pathogen) or focused on overall disease severity. To dissect which fungal function is most influenced by leaf maturity and how, we propose to measure a set of elementary traits underlying components of aggressiveness.

Foliar pathogens of woody plants are good biological models to study the influence of leaf age on disease development, because they can infect leaves at various levels of maturity on the same host plant (Cellerino et al., 1978; Sharma et al., 1980; Turechek and Stevenson, 1998). Poplar is particularly well suited for studying the effect of leaf age on pathogens, since poplar plants grown from cuttings in a glasshouse produce new leaves at a constant rate, giving access to a wide range of leaves of increasing maturity on the same plant (Larson and Isebrands, 1971). These authors introduced the concept of leaf plastochron index (LPI) to refer to a position of a leaf along a poplar shoot. The youngest fully expanded leaf corresponds to LPI 1. Older leaves are numbered with increasing LPIs, according to their position along the shoot. In most experimental conditions the sink to source transition in poplar is established around LPI 6 (Coleman, 1986), which thus defines the first mature leaf. The effect of leaf maturity on the development of poplar pathogens (Marssonina brunnea, Melampsora medusae, and M. larici-populina) has been widely studied (Cellerino et al., 1978; Sharma et al., 1980; Coleman, 1986; Giorcelli et al., 1996). In most of these experiments, leaves were spray-inoculated with spore suspensions. In these conditions, hundreds of spores compete for infection, and as a result, inoculum density has been shown to influence aggressiveness traits (Giorcelli et al., 1996; Pei et al., 2003; Pariaud et al., 2009a). A way to eliminate any possible interaction among spores and to remove the effect of lesion density on trait variation is to use a single-spore inoculation protocol. Moreover, the single-spore inoculation enables the examination of phenotypic variation at the individual lesion level and not by means of average trait values measured on a cohort of lesions.

The aim of this study was to assess the effect of leaf age, and the associated content in nutrients and defense compounds, on the variation in aggressiveness traits in the poplar rust fungus M. larici-populina. We quantified trait variation along the leaf maturity gradient using the same fungal genotype to establish a so-called reaction norm (Gavrilets and Scheiner, 1993). We measured the effect of LPI using a single-spore inoculation method for a suite of aggressiveness and morphological traits: infection efficiency, latent period, uredinia size, sporulation rate, sporulation capacity, in planta mycelium quantity, and urediniospore volume. In a first step, we examined the differences in pathogen development along a leaf maturity gradient in order to study the variation in fungal traits and to assess the correlations between them. In a second step, we estimated the influence of the most important leaf compounds (sugars, total carbon and nitrogen, and phenolics) on the variation in aggressiveness traits.

\section{MATERIALS AND METHODS}

\section{Plant and Fungal Material, and Experimental Design}

Characterization of quantitative traits was performed on excised leaf disks of Populus deltoides $\times$ P. nigra 'Robusta'. Poplar plants were grown from dormant cuttings in 10 -L pots containing a sand-peat $(1: 1, \mathrm{v} / \mathrm{v})$ mixture, with an initial fertilization of $3.5 \mathrm{~g} \mathrm{~L}^{-1} \mathrm{CaCO} 3$ and $6 \mathrm{~g} \mathrm{~L}^{-1}$ of slow release $13-13-13 \mathrm{~N}-\mathrm{P}-\mathrm{K}$ fertilizer (Nutricote ${ }^{\circledR}$ T100, Fertil). The plants were grown in a growth chamber regulated at $20 / 22^{\circ} \mathrm{C}$ (night/day temperatures) and $16 \mathrm{~h}$ photoperiod in order to ensure optimal growth, and were watered daily with deionized water. After four months, plants were about $1.2 \mathrm{~m}$ high and exhibited 25 to 30 fully expanded leaves.

On the same day, we harvested eight leaves of different maturity (LPI 6, 8, 10, 12, 14, 16, 18, and 20) from each of three plants (total of 24 leaves). From each poplar leaf, 24 disks (12-mm-diameter) were excised, and placed in flotation, each in a different cell culture plate. Each plate consists in 24 wells and thus contained one sample (leaf disk) of all leaves. 
A plate thus corresponded to a complete statistical block: Twentyfour replicate plates were prepared, each containing the 24 combinations of LPI and plants. Within plates, the positions of the samples were randomized (Supplementary Figure S1). After disk excision, the remaining part of each leaf (about half a leaf) was stored individually in an aluminum wrapper at $-80^{\circ} \mathrm{C}$ for sugar, nitrogen, carbon, and phenolic compound quantification. We hereafter refer to this plant material as "uninfected leaves."

Because the single-spore inoculation protocol is highly time-consuming, we were not able to inoculate more than six plates per day. Inoculations of the 24 plates were thus distributed over four consecutive days (each inoculation day formed a series of six plates). In other words, all leaf disks were excised the same day (day 1), but inoculated after 0 to 3 days depending on the series they belonged to.

Our experimental design (randomized complete block design) thus consisted in three experimental factors: LPI, plant and day of inoculation. Given an infection efficiency of approximately 25\%, we expected six replicates per combination.

\section{Single-Spore Inoculation Protocol}

Single-spore inoculation was performed with pure M. laricipopulina isolate 98AG31. This isolate was collected in 1998 in Moÿ-de-l'Aisne (France) on Populus trichocarpa $x$ Populus deltoides 'Beaupré leaves and serves since as a reference isolate (Duplessis et al., 2011). Urediniospores were cryopreserved at $-80^{\circ} \mathrm{C}$ at Institut National de la Recherche Agronomique Nancy (France) and multiplied on fresh poplar leaf disks before inoculation to maximize their germination and infection rates (Pei et al., 2002). To this aim, $1 \mathrm{mg}$ of urediniospores was dispersed in $200 \mu \mathrm{L}$ of water-agar $\left(0.1 \mathrm{~g} \mathrm{~L}^{-1}\right)$. The resulting urediniospore suspension was applied as $2 \mu \mathrm{L}$ droplets on the abaxial surface of two 30-mm-diameter leaf disks of 'Robusta'. Leaf disks were incubated by floating on deionized water in six-well polystyrene cell culture plates, with the abaxial surface upside, at $19 \pm 1^{\circ} \mathrm{C}$, and under continuous fluorescent light $\left(25 \mu \mathrm{mol} \mathrm{m} \mathrm{m}^{-2} \mathrm{~s}^{-1}\right)$. After a 10 to 13 days incubation period, the sporulating leaf disks were gently tapped over a microscope slide to release and collect urediniospores.

A germination test was performed just before inoculation to ensure the quality of urediniospores used. To this aim, a few hundred urediniospores were dispersed on the surface of a Petri dish containing agar $\left(20 \mathrm{~g} \mathrm{~L} \mathrm{~L}^{-1}\right)$. After an overnight incubation at $19 \pm 1^{\circ} \mathrm{C}$, the ratio of germinated/total urediniospores was evaluated under a light microscope $(100 \times$ magnification) to determine the germination rate. This test ensured that nearly all spores used for the experiment were physiologically able to germinate (mean germination rate of 93\%).

For single-spore inoculation leaf disks were inoculated one by one. One single urediniospore was picked with a human eyelash under a stereomicroscope (63x magnification) and deposited into a $5 \mu \mathrm{L}$ water-agar droplet placed at the center of the poplar leaf disk (abaxial surface). After inoculation the plates were incubated for 13 days in the same conditions as described above.

\section{Quantitative Trait Measurements}

Several aggressiveness traits classically studied in plantpathogen interactions were measured: infection efficiency, latent period, uredinia size, sporulation rate, and sporulation capacity (Lannou, 2012). In addition, we measured in planta mycelium quantity, which is less commonly studied.

- Infection efficiency is commonly defined as the probability that a urediniospore deposited on a receptive host surface produces a lesion (Lannou, 2012).

- Latent period is defined as the time interval between infection and the onset of sporulation from that infection (Lannou, 2012).

- Uredinia size is defined as the surface area that produces the urediniospores (Kolmer and Leonard, 1986; Robert et al., 2004) and has been suggested to be an indication of the pathogen capacity for host tissue colonization (Pariaud et al., 2009b; Lannou, 2012). However, the uredinia size is not a proxy of tissue colonization by M. larici-populina, as no necrosis is observed. Unlike necrotrophic pathogens, the only visual symptom is the opening in the leaf epidermis, through which urediniospores are extruded.

- Sporulation rate is defined as the number of urediniospores produced by the uredinia per unit of time (Leonard, 1969; Clifford and Clothier, 1974; Kardin and Groth, 1989): sporulation rate $=$ urediniospore number $/(13$ - latent period $)$.

- Sporulation capacity is defined as the amount of spores produced per unit area of sporulating tissue per unit of time (Robert et al., 2002; Dowkiw et al., 2003). It characterizes the flux of spores extruded by the uredinia and is calculated as the ratio between sporulation rate and uredinia size.

- Mycelium quantity which gives a proxy of the fungal colonization in planta is assessed using $\mathrm{qPCR}$ analysis on leaf disks (see below).

Beyond these six quantitative aggressiveness traits, we also measured a morphological trait:

- Volume of urediniospores was computed from length and width of urediniospores obtained from image analysis (see below).

We monitored the infections for 13 days, allowing measurements of latent period and infection efficiency. Emergence of uredinia was scored twice a day, at 9 a.m. and at 4 p.m. Infection efficiency was assessed for each leaf disk and was recorded as a binary variable: 1 if the deposited urediniospore produced one uredinia, 0 if not.

At day 13 (end of the monitoring period), leaf disks were harvested. We harvested all infected leaf disks (total: 158 samples out of 576 leaf disks inoculated, mean infection efficiency of $27.4 \%$ ), giving from 4 to 12 biological replicates per LPI and per plant (mean: 6.58 replicates). The first step of the harvest consisted in separating the urediniospores produced 
from the leaf disk. Infected leaf disks were placed in $2 \mathrm{~mL}$ Eppendorf tubes with $2 \mathrm{~mL}$ of Isoton ${ }^{\circledR}$ II isotonic buffered diluent (Beckman Coulter). The tubes were vigorously shaken for $20 \mathrm{~s}$ at $4 \mathrm{~m} \mathrm{~s}^{-1}$ on a MP FastPrep ${ }^{\circledR}-24$ homogenizer, in order to release all urediniospores from the uredinia. The second step consisted in measuring the size of the uredinia (or lesion size): photographs of the spore-free uredinia were taken under a stereomicroscope $(25 \times$ magnification) coupled to a digital camera. Pictures were analyzed using ImageJ version $1.5 \mathrm{i}$ with a dedicated script (Supplementary Note S1). To measure the number of yellow pixels corresponding to the sporulating area, the Hue Saturation and Brightness was used: pixels with a hue under $37^{\circ}$ were counted. Last, infected leaf disks were stored at $-80^{\circ} \mathrm{C}$ and lyophilized before DNA extraction.

Measurements of both sporulation rate and urediniospore morphological parameters were performed on the urediniospore suspensions using an Occhio $^{\circledR}$ Flowcell FC200+ optical morphogranulometer, which allows simultaneous particle counting and image analysis of the particles. Before counting, urediniospore suspensions were vortexed to disperse clusters of urediniospores. For each sample, counting was performed using the following settings: $0.15 \mathrm{~mL}$ of priming, $0.85 \mathrm{~mL}$ of volume analysis, and $7 \%$ of volume sampling (i.e., $7 \%$ within $0.85 \mathrm{~mL}$ were really analyzed, evenly distributed along the analyzed volume). Two filters parameter values were applied:

1. For urediniospore counting: bluntness between 0.6 and 1 ; length of ellipse minor axis between 20 and $60 \mu \mathrm{m}$; ellipse elongation between 0.18 and 0.79 ; and ISO eccentricity higher than 0.15 ,

2. For urediniospore size measurement: bluntness between 0.6 and 1; inertia Feret elongation between 0.25 and 1; ISO eccentricity higher than 0.1 ; minimum Feret diameter between 13.5 and $70 \mu \mathrm{m}$; and Feret diameter length between 23 and $200 \mu \mathrm{m}$.

These filters were applied on the raw data to compute sporulation rate expressed in spores per day, length, and width of urediniospores produced by each uredinia. The volume of urediniospores was computed following the formula of the volume of an ellipsoid (Philibert et al., 2011):

$$
\text { Spore volume }=\frac{4}{3} \pi \times \frac{\text { length }}{2} \times\left(\frac{\text { width }}{2}\right)^{2}
$$

\section{DNA Extraction and qPCR Quantitation of in planta Mycelium}

DNA was extracted from infected poplar leaf disks using the DNeasy96 ${ }^{\circledR}$ DNA plant kit (Qiagen). The Frozen Plant Tissue protocol (DNeasy 96 Plant Handbook, June 2015) was followed except that $201-\mathrm{mm}$-diameter glass beads were added to one 3-mm-diameter tungsten carbide bead in the $2 \mathrm{~mL}$ Eppendorf tubes. The 158 samples were randomly processed in two DNA extraction plates, which were stored at $4^{\circ} \mathrm{C}$ until qPCR analysis.

In order to correct for amplification differences among qPCR, three technical replicates were performed.
In order to quantify M. larici-populina mycelium in planta, quantitative Taqman ${ }^{\circledR}$ PCR was performed using new primers and probe designed for the rDNA ITS (Internal Transcribed Spacer). These primers and probe allowed a significant gain in sensitivity compared to a previously used primer pair, which was designed for a single-copy gene (Guinet et al., 2016). Reagent mix contained $3.1 \mu \mathrm{L}$ of pure water, $6.5 \mu \mathrm{L}$ of Mastermix ${ }^{\circledR}$, $0.13 \mu \mathrm{L}$ of primer ITS-Mlp-F (Primer sequence $5^{\prime}-3^{\prime}$ : TGACTCTTTGTATAAACCATTACCC), $0.13 \mu \mathrm{L}$ of primer ITS-Mlp-R (TCAAAGTTGCCTTTGAGATACG), and $0.13 \mu \mathrm{L}$ of probe ITS-Mlp-P (6-FAM-TGCATTGTGGCCCGTCAAAABHQ1) per sample. A volume of $3 \mu \mathrm{L}$ of extracted DNA was added to $10 \mu \mathrm{L}$ of reagent mix. Standard samples consisted of calibrated quantities of plasmid DNA containing the cloned ITS amplicon. Two series of eight 10-fold dilutions of plasmid DNA (from $1.47 \times 10^{7}$ to $1.47 \mathrm{fg}_{\mathrm{ML}}^{-1}$ ) were included in each qPCR run to set a standard curve. Quantitative PCR runs were performed on a QuantStudio 6 Flex (Life Technologies) machine and consisted in $10 \mathrm{~min}$ at $95^{\circ} \mathrm{C}$ followed by 45 cycles of $15 \mathrm{~s}$ at $95^{\circ} \mathrm{C}$ and $45 \mathrm{~s}$ at $62^{\circ} \mathrm{C}$. DNA quantity was analyzed using the QuantStudio $^{\mathrm{TM}}$ Real-time PCR software (version 1.1).

\section{Leaf Content Measurements: Total Nitrogen and Total Carbon Contents, Sugars, Phenolic Compounds}

All leaf content measurements were conducted on the uninfected leaves, stored at $-80^{\circ} \mathrm{C}$ just after disk excision.

Total nitrogen and total carbon concentrations in uninfected leaves were analyzed using an elemental analyser (Thermo Quest, Type NCS 2500). Measurements, data analysis, data record, and weighing record (Mettler Tolédo MT5 microbalance) were performed using the EAGER 200 software.

Soluble sugars were extracted from $20 \mathrm{mg}$ of dry leaf powder with $1 \mathrm{~mL}$ of $70 \%(\mathrm{v} / \mathrm{v})$ methanol/water for $10 \mathrm{~min}$ and then centrifuged at $17,000 \mathrm{~g}$ for $5 \mathrm{~min}$ at $4^{\circ} \mathrm{C}$. This step was repeated twice and the resulting supernatants were pooled together. The supernatants, containing soluble sugars, were dried overnight with a vacuum evaporator (Maxi-Dry Plus; Hetomodel DW1, 0-110, Heto-HOLTEN A/S) to eliminate methanol. Dried extracts were rehydrated in $1.5 \mathrm{~mL}$ of distilled water, dissolved by sonication and then filtered at $0.2 \mu \mathrm{m}$ (Acrodisc ${ }^{\circledR}$ Supor ${ }^{\circledR}$ filter; $0.2 \mu \mathrm{m}$, Van Waters Rogers). Undiluted aliquots of $20 \mu \mathrm{L}$ were injected into a high-pressure liquid chromatography system (Dionex ${ }^{\mathrm{TM}}$ ICS-5000 + HPLC $^{\mathrm{TM}}$ ) to determine soluble sugar composition. Soluble sugar separation was achieved on a Dionex ${ }^{\mathrm{TM}}$ CarboPac $^{\mathrm{TM}}$ SA10 IC separation column $(6 \mu \mathrm{m}$ $250 \times 4 \mathrm{~mm}$; Thermo Scientific $\left.{ }^{\mathrm{TM}}\right)$. The flow rate was maintained at $1 \mathrm{~mL} \mathrm{~min}-1$, and the column temperature at $40^{\circ} \mathrm{C}$. Individual carbohydrates were eluted from 0 to $30 \mathrm{~min}$ after injection. The sugar peaks were detected by light scattering (Sedex 45 ELSD system, Seder) then identified according to retention time and commercial standards. Sucrose, glucose, and fructose concentrations (\% of dry weight) were determined by peak height and standard calibrations.

Phenolics were extracted from $30 \mathrm{mg}$ dry leaf powder using the method described by Royer et al. (2013). Total phenolic content 
was determined according to the Folin-Ciocalteu method. Briefly, each extract was diluted 1:16 in distilled water, and $20 \mu \mathrm{L}$ of each diluted extract was distributed in a 96-well plate. Then $100 \mu \mathrm{L}$ of Folin-Ciocalteu's reagent (Sigma), diluted 1:10 in distilled water, and $80 \mu \mathrm{L}$ of $7.5 \%$ sodium carbonate $(\mathrm{w} / \mathrm{v})$ were added. The microplate was incubated $30 \mathrm{~min}$ at $25^{\circ} \mathrm{C}$ and shaken $5 \mathrm{~s}$ every $10 \mathrm{~min}$. Absorbance of the sample was measured with a microplate-reader (SynergyHT, BioTek) at $760 \mathrm{~nm}$. Quantification of total phenolics was performed according to a calibration curve of gallic acid, and the results were expressed as milligram of gallic acid equivalent per gram of leaf dry weight.

In addition to total phenolic content, the concentration of major phenolics was analyzed on ultra-high performance liquid chromatography (U-HPLC) system (Shimadzu) equipped with a photo diode array detector (DAD) and a mass spectrometer. Each sample $(3 \mu \mathrm{l})$ was separated on a C18 kinetex $(100 \mathrm{~mm} \times 2.1 \mathrm{~mm})$ column (Phenomenex). The mobile phase consisted in $0.1 \%$ formic acid in ultra-pure water (solvent A) and $0.1 \%$ formic acid in methanol (solvent B). The molecules were eluted with a flow rate of $300 \mu \mathrm{L} \mathrm{min}{ }^{-1}$, through a gradient elution from 1 to $50 \% \mathrm{~B}$ for $10 \mathrm{~min}$, then to $99 \% \mathrm{~B}$ in $3 \mathrm{~min}$, which was maintained for three additional minutes. The column was then re-equilibrated to $1 \% \mathrm{~B}$ for $4 \mathrm{~min}$ prior to the next run. Mass spectrometry analysis was carried out in ESI positive and negative modes. Quantification was performed by measuring the area under each peak at 280, 320, or $350 \mathrm{~nm}$, depending on the lambda max of each molecule. Due to coeluting signal at $280 \mathrm{~nm}$, catechin quantification was realized by measuring the area under peak at $m / z 289$, corresponding to the $[\mathrm{M}-\mathrm{H}]-$ of the molecule. Experimental exact masses and MS fragments were compared to metabolomics databases $\left(\right.$ Respect $^{1}$; MassBank $\left.{ }^{2}, \mathrm{DNP}^{3}\right)$ and data available in the literature in order to identify the nature of the metabolites. Sixteen phenolic compounds were identified. According to their structure, these 16 metabolites could be grouped into four phenolic subclasses, which are Flavan-3-ols (catechin, proanthocyanidin dimer), hydroxycinnamic acid esters (chlorogenic acid, caffeoyl shikimate, caffeoyl glucose isomers $(\times 2)$, coumaroyl glucose isomers $(\times 2)$ ), flavonols (rutin, quercitrin), and salicinoids (salicine, salicortin, acetylsalicortin, homaloside D, tremulacin, and a putative populoside) (Supplementary Figure S2).

\section{Data Analysis}

Principal component analyses (PCA) and linear models were used to explore correlations between phenotypic traits. Each independent phenotypic trait was analyzed separately using a generalized linear model evaluating effects of LPI, plant, day of inoculation, and the interaction between LPI and plant. To normalize the error, infection efficiency was analyzed with a binomial error family; latent period and sporulation capacity with a Gamma error family; uredinia size, spore volume, sporulation rate, and mycelium quantity with a Gaussian error family; and uredinia size and sporulation rate were square root

\footnotetext{
${ }^{1}$ http://spectra.psc.riken.jp/

${ }^{2}$ http://massbank.jp/

${ }^{3}$ http://dnp.chemnetbase.com/
}

transformed. An analysis of variance (ANOVA) with an alpha error $<5 \%$ was performed on each model. Differences of means were checked with Tukey tests. PCA on leaf characteristics was performed to explore their variations. Spearman correlation coefficients between phenotypic traits and leaf content were computed on mean phenotypic traits per LPI and plant based on 4 to 12 replicates. Spearman correlation coefficients' associated $p$-values were adjusted with a False-Discovery-Rate correction for multiple tests. All data analyses were performed using $\mathrm{R}$ version 3.2.5 (see details in the RMARKDOWN document, Supplementary Note S2).

\section{RESULTS}

\section{Variation in M. larici-populina Quantitative Traits}

To describe the variation in quantitative traits of $M$. laricipopulina in this experiment, a PCA was performed on individual trait measurements assessed for each infected leaf disk (latent period, uredinia size, sporulation rate, sporulation capacity, mycelium quantity, and mean volume of produced spores). Infection efficiency cannot be included in the PCA analysis because it is set to one for infected leaf disk. Experimental factors (LPI, plant harvested, and day of inoculation) were considered as supplementary variables. The first principal component (PC1) explained $31 \%$ of the total inertia and was related to uredinia size, sporulation rate and to a lesser extent to sporulation capacity (Figure 1). PC2 and PC3 accounted for almost 20\% and 17\% of the total variance, respectively. PC2 was related to latent period which varied independently from uredinia size and sporulation rate, and PC3 was related to spore volume. However, there was large dispersal of individual measures that remained unexplained by experimental factors (Supplementary Note S2).

In accordance with PCA analysis, LPI was the main structuring variable in GLM analyses and had a significant effect on uredinia size $\left(P_{\text {adjusted }}=1.22 \times 10^{-21}\right)$, sporulation rate $\left(P_{\text {adjusted }}=7.67 \times 10^{-15}\right)$, and sporulation capacity $\left(P_{\text {adjusted }}=1.40 \times 10^{-05}\right)($ Table 1$)$. Uredinia size and sporulation rate increased with the LPI, while sporulation capacity decreased (Figure 2). Uredinia size exhibited a threefold increase (from 0.272 to $0.737 \mathrm{~mm}^{2}$ ) and sporulation rate a twofold increase (from 333 to 611 urediniospores day ${ }^{-1}$ ) from LPI 6 to 20 (Figure 2). Sporulation capacity was reduced more than twofold from LPI 6 to 20 (from 12,420 to 5,800 urediniospores day $^{-1} \mathrm{~mm}^{-2}$ ) (Figure 2). The sporulation rate was also significantly, although less strongly, explained by the plant used for the experiment $\left(P_{\text {adjusted }}=4.63 \times 10^{-05}\right)$. Sporulation rate was significantly higher on plant No. 3 (mean rate $=526$ urediniospores $\mathrm{day}^{-1}$ ) and lower on plant No. 2 (mean rate $=396$ urediniospores day ${ }^{-1}$ ) (Figure 2), but no interaction was found between plant and LPI (Table 1). Other fungal traits varied but not in accordance with LPI, plant and day (Supplementary Figure S3).

We found no significant effect of the day of inoculation on any aggressiveness traits. As there was no difference in fungal traits across days of inoculation, we assumed that the incubation 


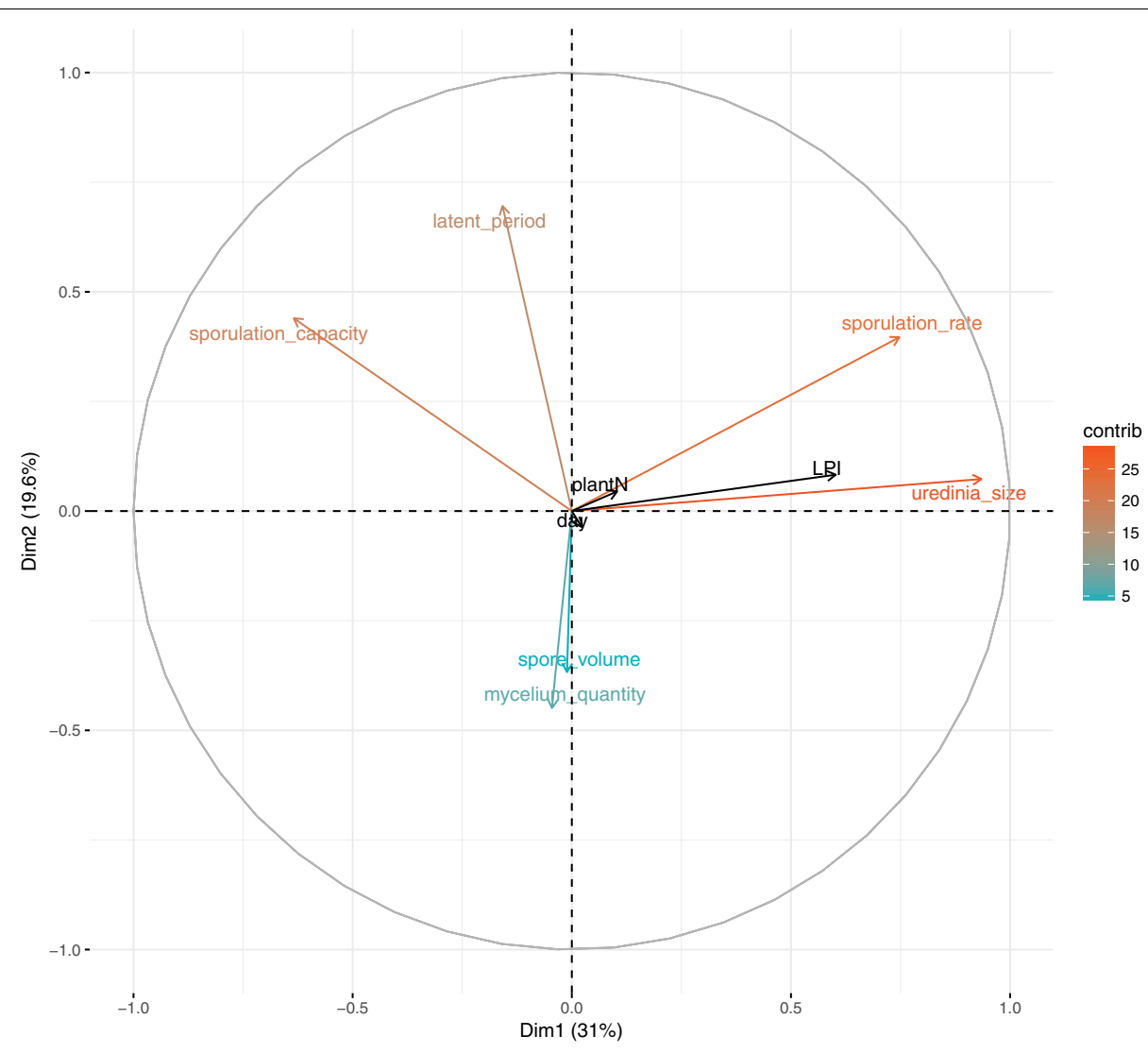

FIGURE 1 | Principal component analysis of fungal phenotypic traits (latent period, uredinia size, sporulation rate, sporulation capacity, spore volume, and mycelium quantity, measured on 4 to 12 replicates per LPI and per plant); LPI, plant and day are supplementary variables. Colors correspond to variables' contribution to the first and second axes.

TABLE 1 | Summary of the analysis of variance of each experimental variable in generalized linear models (Chi-square test probability adjusted with a False-Discovery-Rate correction for multiple tests).

\begin{tabular}{lcccc}
\hline Traits & LPI & Plant & LPI:Plant & Day \\
\hline Infection efficiency & 0.459 & 0.661 & 0.346 & 0.165 \\
Latent period & 0.720 & 0.165 & 0.720 & 0.661 \\
Uredinia size & $\mathbf{1 . 2 2} \times \mathbf{1 0}^{-\mathbf{2 1}}$ & 0.092 & 0.458 & 0.346 \\
Spore volume & 0.980 & 0.362 & 0.720 & 0.720 \\
Sporulation rate & $\mathbf{7 . 6 7} \times \mathbf{1 0}^{-\mathbf{1 5}}$ & $\mathbf{4 . 6 3 \times \mathbf { 1 0 } ^ { - 0 5 }}$ & 0.497 & 0.090 \\
Sporulation capacity & $\mathbf{1 . 4 0 \times \mathbf { 1 0 } ^ { - 0 5 }}$ & 0.379 & 0.720 & 0.092 \\
Mycelium quantity & 0.866 & 0.980 & 0.996 & 0.720
\end{tabular}

$P$-values $<0.05$ are indicated in bold characters.

conditions during the pre-inoculation period described above kept the leaf disks in their initial physiological state.

\section{Variation in Leaf Content Characteristics}

To assess leaf content variations among the range of leaf maturity, a PCA was performed on leaf characteristics (Figure 3). Leaf characteristics were measured on one uninfected half-leaf of each plant and each LPI, so only plant and LPI supplementary variables were taken into account. PC1 explained almost 53\% of the total inertia and was highly related to sugars (sucrose, fructose, glucose) and phenolic compounds (total phenolics, and all the phenolic subclasses) variations. Explaining $18 \%$ and $12 \%$ of the inertia, PC2 and PC3 were associated to sucrose and nitrogen, respectively. The total carbon content of leaves did not vary among the range of leaf maturity studied. Leaf maturity, characterized by the content in phenolic compounds, appeared to structure more leaf characteristics than plant did.

\section{Correlation of Fungal Phenotypic Trait Variation and Leaf Content Characteristics}

To assess how fungal and leaf data were correlated, pairwise Spearman's correlation coefficients and their adjusted $p$-values were calculated.

Spearman's coefficient for uredinia size and both sporulation rate and sporulation capacity were highly significant (rhô $=0.87$ and $-0.73, P_{\text {adjusted }}=3.37 \times 10^{-6}$ and $4.66 \times 10^{-4}$, respectively) (Figure 4), which is consistent with PCA results on individual traits. No other Spearman's coefficient appeared significant between fungal traits.

Spearman coefficients showed a negative correlation between total phenolic content and uredinia size and sporulation rate 

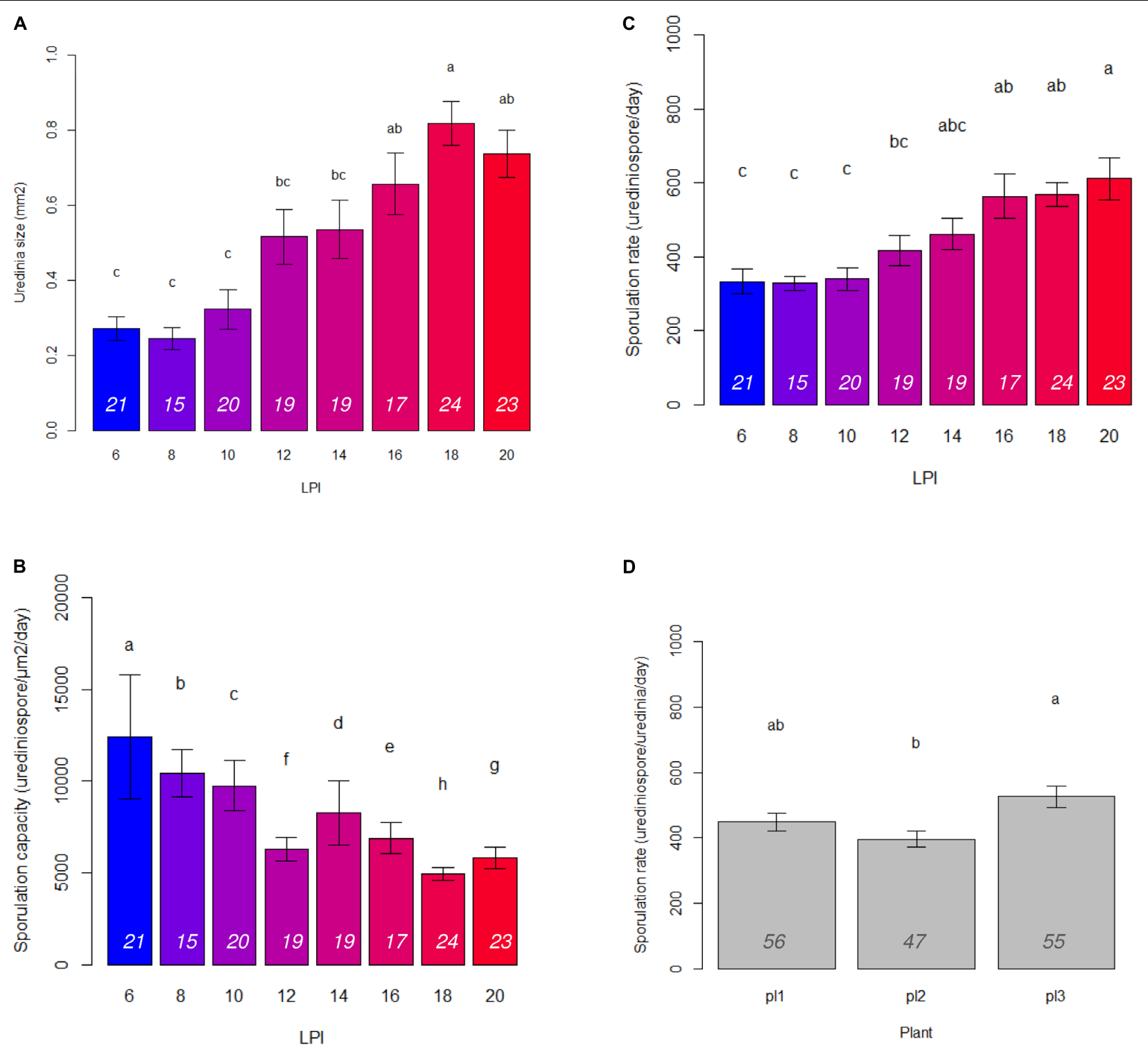

D

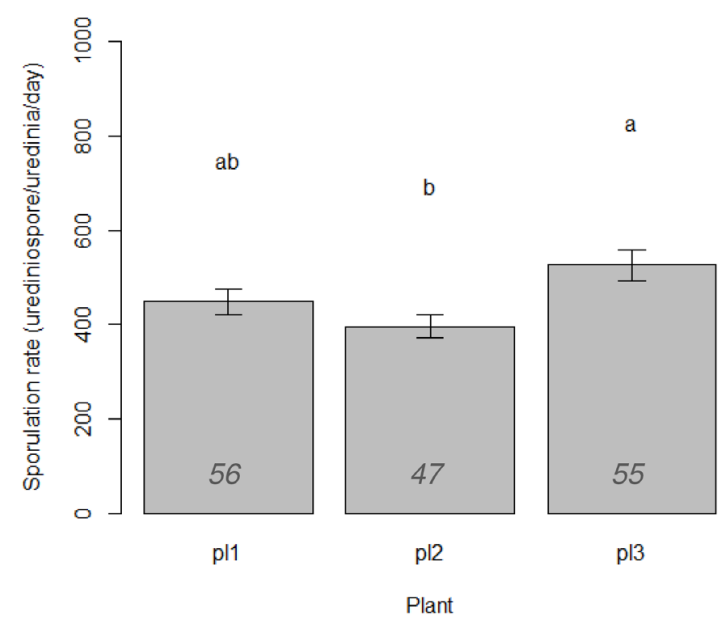

FIGURE 2 | Barplots of means and standard error calculated on three plants and 15 to 24 replicates per LPI (italic numbers at the bottom of the bars) of (A) uredinia size, (B) sporulation capacity, and (C) sporulation rate for each LPI; and (D) sporulation rate for each plant. Letters correspond to Tukey test results.

(rhô $=-0.77$ and $-0.61, P_{\text {adjusted }}=1.62 \times 10^{-4}$ and $1.07 \times 10^{-2}$, respectively), as well as a negative correlation between hydroxycinnamic acid esters content and uredinia size and sporulation rate ( rhô $=-0.83$ and $-0.68, P_{\text {adjusted }}=2.25 \times 10^{-5}$ and $1.85 \times 10^{-3}$, respectively), and a positive correlation between hydroxycinnamic acid esters content and sporulation capacity $\left(\right.$ rhô $\left.=0.68, P_{\text {adjusted }}=1.85 \times 10^{-3}\right)$ (Figures 4, 5). Three of the four phenolic subclasses (salicinoids, hydroxycinnamic acid esters, and flavonols) showed the same trends as total phenolic content, with the exception that the correlation between sporulation rate and sporulation capacity, on one side, and salicinoids and flavonols, on the other side, were not significant $\left(P_{\text {adjusted }}>0.05\right)$. In accordance with the PCA on leaf characteristics, all sugar contents were correlated with each other.
However, no correlation was found between fungal phenotypic traits and sugars, carbon and nitrogen contents. Salicinoids, flavonols, and hydroxycinnamic acid esters were all positively correlated to each other. Flavan-3-ols were, however, negatively correlated to the three other phenolic subclasses, although this was only significant with flavonols.

\section{DISCUSSION}

In this study, we analyzed the variation in seven aggressiveness and morphological traits of the poplar rust fungus when inoculated on poplar leaves of varying maturity levels. A single-spore inoculation technique was used, which enabled 


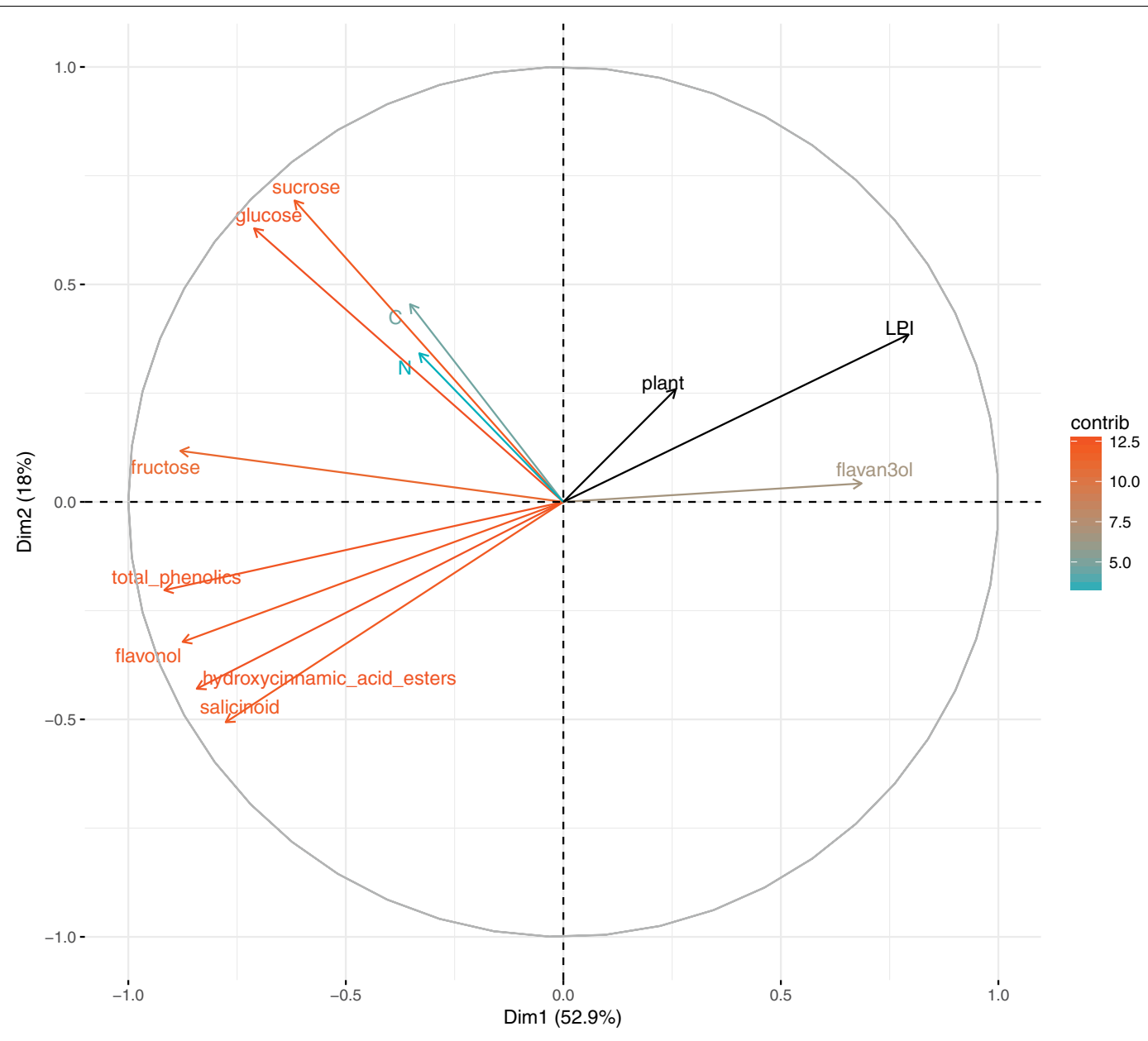

FIGURE 3 | Principal component analysis on leaf chemical content (carbon, nitrogen, glucose, fructose, sucrose, total phenolics, flavan-3-ols, hydroxycinnamic acid esters, flavonols, and salicinoids), LPI and plant are supplementary variables. Colors correspond to variables' contribution to the first and second axes.

us to study trait variance and covariance at the individual lesion level. Applying this method, we aimed at assessing the effect of leaf maturity on aggressiveness traits of M. larici-populina. In a second step, we explored correlations between the variation in fungal phenotypic traits and physiological features of the leaves in order to determine whether variation in phenotypic traits was influenced by nutritive or defensive compounds of leaves.

\section{Effect of Leaf Maturity on Aggressiveness and Morphological Traits}

Regarding the variation in aggressiveness traits, our first result was a positive correlation between uredinia size and sporulation rate, whereby both increased with leaf maturity. That is, on more mature leaves, uredinia were wider with a higher production of urediniospores. Some authors found that the breaking down of sporulation rate into uredinia size and sporulation capacity enabled to explain differences in aggressiveness levels: an increase in sporulation rate can either be due to wider uredinia or higher sporulation capacity (flux of urediniospores) (Pariaud et al., $2009 b$ ). In our study, we found that the increase in sporulation rate is linked to wider uredinia, but not to higher sporulation capacity. The correlation between uredinia size and sporulation rate has already been identified by several authors, including Dowkiw et al. (2003) on poplar rust, Pei et al. (2002) on willow rust and Robert et al. (2002) on wheat leaf rust. Based on our data, we add to this that sporulation capacity is not necessarily even as assumed by Robert et al. (2002). Indeed, sporulation capacity significantly decreased with leaf maturity, in contrast to uredinia size and sporulation rate. Even if the flux of urediniospores was lower on mature leaves, the amount of urediniospores produced was higher, leading to an increased aggressiveness on mature leaves compared to younger ones.

In this study, we also quantified individual variation in mycelium quantity through qPCR analysis. This trait has received little attention as a component of aggressiveness, mostly for methodological reasons. However, it can serve to measure the relative investment in sporulation vs. mycelium growth, the two main functions to which host extracted resources are allocated (Gilchrist et al., 2006; Yegorov et al., 2017). Notably, the quantity of in planta mycelium did not vary along the leaf maturity gradient and was correlated to neither sporulation rate nor lesion size. Hence, lesion size does not equate to mycelium 


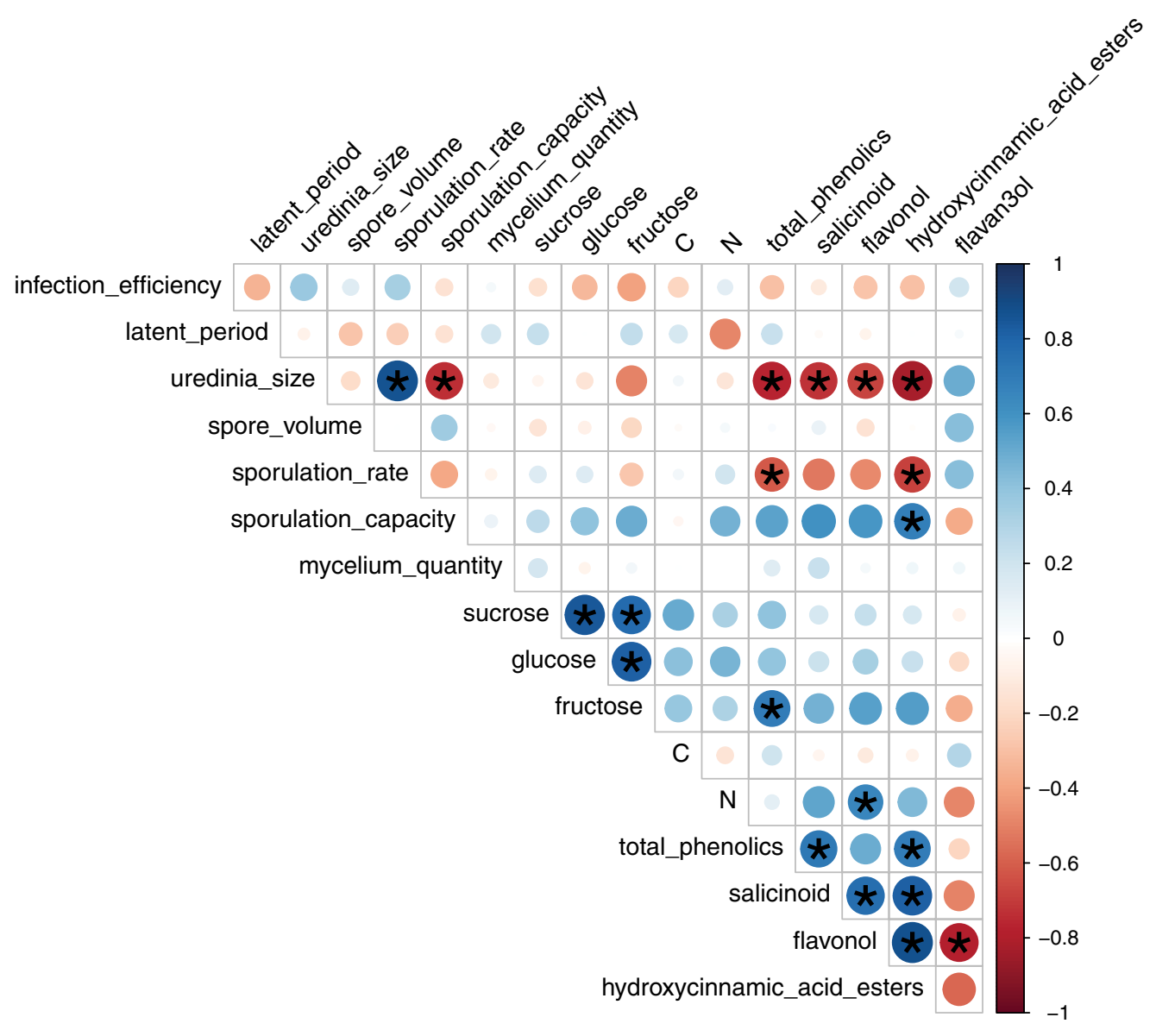

FIGURE 4 | Pairwise Spearman correlation coefficients between leaf chemical content and mean fungal phenotypic traits per LPI and plant based on 4 to 12 replicates. Colors correspond to the sign and dot size to the strength of the correlation. Stars indicate significant correlations according to Student $t$-test adjusted with a False-Discovery-Rate correction for multiple tests.

quantity. This makes sense as for biotrophic fungi like rusts, lesion (uredinia) size is only the size of the aperture through which urediniospores are extruded, and may thus not be directly linked to the size of the area within a leaf that is colonized by the mycelium.

Neither leaf maturity, nor the two other experimental factors (plant and day of inoculation) had an effect on latent period, infection efficiency, mycelium quantity and spore volume, even though substantial variances were observed for those traits. The latent period and spore volume varied independently from each other and independently from the main structuring traits (uredinia size and sporulation rate). Variation in latent period and spore volume might have been affected by an unidentified environmental factor. Even though inoculated leaf disks were incubated under controlled conditions of temperature and light, micro-scale variation may have occurred for these crucial parameters influencing fungal development and infection dynamics (Cochrane, 1959; Rotem et al., 1978; Trapero-Casas and Kaiser, 1992; Turechek and Stevenson, 1998; Turechek et al., 2001; Pap et al., 2013). Moreover, the initial position of the disk in the leaf might have influenced infection outcome
(Katsuya and Green, 1967; Johnston et al., 2016). Singlespore inoculation is more prone to inter-replicate variance, in contrast to spray-inoculation which buffers micro-environmental variations. Apart from this drawback, this method allowed us to accurately disentangle the variation in each trait and to properly assess putative correlations. In our study, the dissection of the infection process into elementary traits enabled us to assess that the leaf maturity gradient only influences the sporulation function.

\section{Disentangling Nutritive Limitation vs. Defense Reaction Effects}

Two hypotheses were considered to explain variation in uredinia size and sporulation rate. On the one hand, sporulation could be enhanced by a higher nutrient availability in older leaves. On the other hand, higher levels of defense compounds could in contrast prevent fungal development in younger leaves.

Leaf sugars have long been known to be essential to the development of plant pathogenic fungi (Bilgrami and Verma, 1978; Madan and Thind, 1998). Using Populus deltoides leaves 

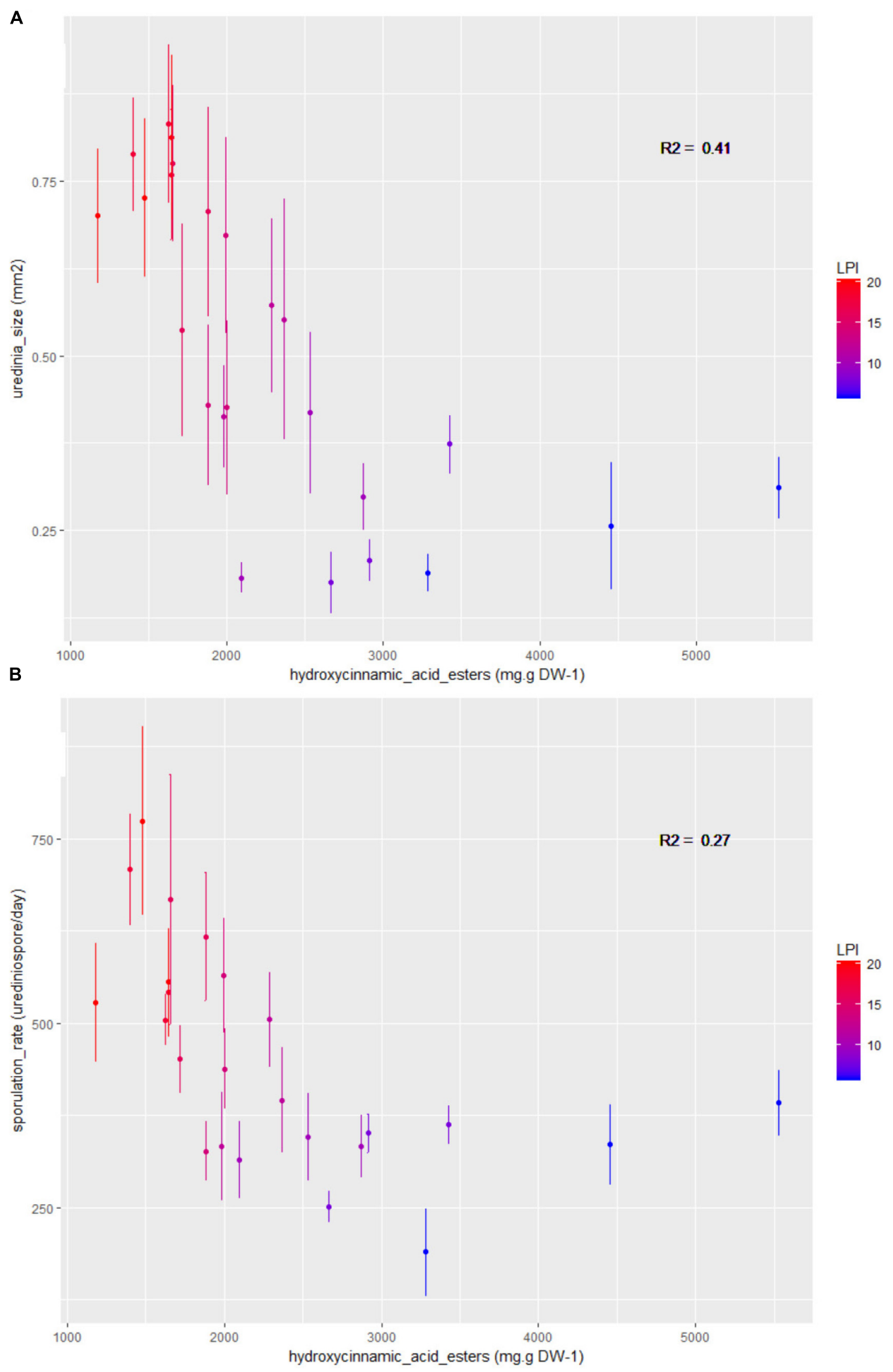

FIGURE $\mathbf{5}$ | Bivariate plots (LPI and plant mean \pm standard error calculated on three plants and 15 to 24 replicates per LPI) showing the variation in (A) uredinia size and (B) sporulation rate according to the phenolic subclass hydroxycinnamic acid esters content. Colors stand for LPI and R2 gives the coefficients of determination. 
inoculated with Melampsora medusae f. sp. deltoidae, Coleman (1986) showed that the maximum level of susceptibility, measured by the number of lesions (uredinia) as a proxy for infection efficiency, is reached when the sugar availability is maximal, i.e., at a LPI of 5. This finding is inconsistent with our study, in which the infection efficiency did not vary with leaf maturity and the two responsive traits (uredinia size and sporulation rate) steadily increased from LPI 6 to 20 . The discrepancy between Coleman (1986) experiments and our results may lie in differences in the growth conditions of poplar plants, which may lead to different levels of nutrient availability. The light quality available in growth chambers has been significantly improved during the last decades. In Coleman (1986), the total sugar content decreased from LPI 6 to 9 (the highest LPI measured). In our experiment, even though sugar contents displayed some variance among leaves, there was no consistent pattern along LPI. Our growth conditions may have led to sugar availability sufficient for steady infection success even at higher LPI. We therefore conclude that leaf sugar content may not be linked to leaf susceptibility per se.

Leaf nitrogen content also plays a role in fungal growth and sporulation (Bilgrami and Verma, 1978; Madan and Thind, 1998). Bilgrami and Verma (1978) stated that nitrogen sources are used by fungi for both structural and metabolic functions. These functions require the assimilation of nitrogen into amino acids, proteins, and some peptides. For example, Jensen and Munk (1997) and Robert et al. (2004) found that higher nitrogen fertilization enhanced barley powdery mildew and wheat leaf rust sporulation. In our experiment, although there was a substantial variance in leaf nitrogen contents [same order of magnitude as in Robert et al. (2004)], this variation did not significantly affect M. larici-populina development. The leaf nitrogen content only slightly correlated with latent period and sporulation capacity (urediniospores produced par day and per area).

Overall, we did not observe consistent variation in nutrient content along the leaf maturity gradient and, moreover, the variation in nutrient content was not correlated with the two responsive traits, uredinia size, and sporulation rate. Consequently, nutrient availability was apparently not the environmental factor best explaining fungal development in our experiment.

By contrast, the defense hypothesis, tested by assessing the constitutive level of phenolics (total and individual) appeared to better explain fungal development related to leaf age. Indeed, the total phenolic content decreased with leaf age and correlated negatively with uredinia size and sporulation rate. Although higher phenolic concentration in younger leaves is common in poplar and other plants (Coleman, 1986; Johnson and Kim, 2005; Royer et al., 2013; Ullah et al., 2017), the correlation between phenolic concentrations and resistance to fungal infection has not been demonstrated. Coleman (1986) and Coleman et al. (1987) did not detect a correlation between leaf phenolic content and the amount of uredinia produced by M. medusae on poplar trees. By contrast, in the same pathosystem, a negative correlation was observed between total phenolic content and urediniospore germination efficiency and hyphae length (Johnson and Kim, 2005). Other studies on black currant (Ribes nigrum) and willow
(Salix myrsinifolia) identified negative correlation between the concentrations of phenolic compounds and development traits of rusts (Hakulinen et al., 1999; Vagiri et al., 2017). By analyzing specific phenolics from poplar leaves, we were able to precisely identify groups of phenolic compounds that correlate with poplar resistance to rust. Among the four phenolic subclasses (from a total of 16 compounds), three subclasses (hydroxycinnamic acid esters, flavonols, and salicinoids) showed the same effect as total phenolic contents and correlated negatively with uredinia size and sporulation rate. The hydroxycinnamic acid ester chlorogenic acid has been shown to correlate to rust resistance in black currant (Vagiri et al., 2017). In addition, several studies highlighted the role of leaf surface flavonoids in the resistance to rust in poplar and birch (Shain and Miller, 1982; Valkama et al., 2005). In our study, we did not address the leaf location (inside or on the surface) of the two flavonols, rutin and quercetrin, however, the fact that they negatively correlate with rust development is in agreement with these previous studies. The only phenolic subclass that did not correlate with resistance to rust was flavan-3-ol which integrates catechin and proanthocyanidin dimers. A role of this phenolic subclass - especially catechin - in resistance to fungal pathogens, including rust fungi, has been proposed in several studies (Brignolas et al., 1998; Hakulinen et al., 1999; Vagiri et al., 2017). Moreover, a recent study on the M. larici-populina - Populus nigra pathosystem demonstrated that catechin is an effective chemical defense compound against rust (Ullah et al., 2017). This demonstration was based on the fact that (i) catechin is strongly induced upon rust infection, (ii) catechin exhibit a strong antifungal activity against $M$. larici-populina at in planta concentration, and (iii) poplar engineered to accumulate high or low levels of catechin were more resistant or more susceptible to rust, respectively. In our study, the concentrations of flavan3-ols increased with leaf age. The same trend was observed in the study by Ullah et al. (2017) (see Supplementary Figure S2). The absence of a correlation between flavan-3-ol concentrations and rust resistance does not indicate that flavan-3-ols are not important factors in leaf rust resistance. Indeed, the compounds were assayed in uninfected leaves; hence reflect constitutive (i.e., non-induced) defense levels. In addition, the concentrations measured in our experiment (from 0.2 to $0.5 \mathrm{mg} . \mathrm{g} \mathrm{DW}^{-1}$ ) were below the concentrations proven to efficiently impair rust development on black poplar leaves (Ullah et al., 2017).

\section{CONCLUSION}

Melampsora larici-populina development after single-spore inoculation onto poplar leaf disks was more constrained by the level of plant constitutive defenses than limited by nutrient availability. For the tested strain, uredinia size and sporulation rate, both components of the sporulation function, were the only traits responsive to reaction norms along a gradient of leaf maturity. This experiment - conducted on a single fungal isolate and on a single plant genotype - can nonetheless serve as a pilot study. We report the correlation of a fungal fitness component (sporulation) to an environmental characteristic imposed by the 
leaf i.e., the constitutive level of phenolic content. In the future, it would be worthwhile to assess the generality of this finding with more fungal isolates and plant genotypes and decipher further the interplay between constitutive and induced defense compound levels. From a broader perspective, it would be interesting to determine the plasticity and heritability of sporulation-related traits to better assess how the complexity of leaf environment shapes the evolutionary trajectory of a plant pathogen.

\section{AUTHOR CONTRIBUTIONS}

$\mathrm{FH}, \mathrm{PF}$, and $\mathrm{BF}$ designed the study. AM conducted the experiment and analyses. MP developed the single-spore inoculation and trait measurement methods. AA developed the qPCR method based on new primers and probe for Melampsora larici-populina in the rDNA ITS (Internal Transcribed Spacer) designed by CG and A-LB. RL conducted leaf phenolics analyses. $\mathrm{AM}$ and $\mathrm{FH}$ wrote the first draft of the manuscript. PF, MP, and $\mathrm{RL}$ contributed to the manuscript. All the authors approved the final version of the manuscript.

\section{FUNDING}

This work was supported by grants from the French National Research Agency (Grant No. ANR-13-BSV7-0011, FunFit

\section{REFERENCES}

Adandonon, A., Regnier, T., and Aveling, T. A. S. (2017). Phenolic content as an indicator of tolerance of cowpea seedlings to Sclerotium rolfsii. Eur. J. Plant Pathol. 149, 245-251. doi: 10.1007/s10658-017-1178-9

Agrios, G. N. (2005). Plant Pathology. New York, NY: Elsevier Academic Press.

Bilgrami, K. S., and Verma, R. N. (1978). Physiology of Fungi. New Delhi: Vikas Publishing House Pvt Ltd.

Boonekamp, P. M. (2012). Are plant diseases too much ignored in the climate change debate? Eur. J. Plant Pathol. 133, 291-294. doi: 10.1007/s10658-0119934-8

Brignolas, F., Lieutier, F., Sauvard, D., Christiansen, E., and Berryman, A. A. (1998). Phenolic predictors for Norway spruce resistance to the bark beetle Ips typographus (Coleoptera: Scolytidae) and an associated fungus, Ceratocystis polonica. Can. J. Forest Res. 28, 720-728. doi: 10.1139/cjfr-285-720

Cellerino, G. P., Anselmi, N., and Pinon, J. (1978). Influence de l'âge des feuilles de peuplier sur la sensibilité à Marssonina brunnea. Eur. J. Forest Pathol. 8, 273-279. doi: 10.1111/j.1439-0329.1978.tb00639.x

Clifford, B. C., and Clothier, R. B. (1974). Physiologic specialization of Puccinia hordei on barley hosts with non-hypersensitive resistance. Trans. Br. Mycol. Soc. 63, 421-430. doi: 10.1016/S0007-1536(74)80088-4

Cochrane, V. W. (1959). Physiology of Fungi. New York, NY: John Wiley \& Sons, Inc.

Coleman, J. S. (1986). Leaf development and leaf stress: increased susceptibility associated with sink-source transition. Tree Physiol. 2, 289-299. doi: 10.1093/ treephys/2.1-2-3.289

Coleman, J. S., Jones, C. G., and Smith, W. H. (1987). The effect of ozone on cottonwood - leaf rust interactions: independence of abiotic stress, genotype, and leaf ontogeny. Can. J. Bot. 65, 949-953. doi: 10.1139/b87-131

Dowkiw, A., Husson, C., Frey, P., Pinon, J., and Bastien, C. (2003). Partial resistance to Melampsora larici-populina leaf rust in hybrid poplars: genetic variability in inoculated excised leaf disk bioassay and relationship with complete resistance. Phytopathology 93, 421-427. doi: 10.1094/PHYTO.2003.93.4.421 project; ANR-11-LABX-0002-01, Cluster of Excellence ARBRE). AM and MP were supported by Ph.D. fellowships from the Region Lorraine and INRA, and from the French Ministry of Education and Research (MESR), respectively.

\section{ACKNOWLEDGMENTS}

Jérémy Pétrowski is acknowledged for his excellent technical help in poplar plant production. We thank Jacqueline Marchand and Carole Antoine (Université de Lorraine, AgroParisTech, INRA, SILVA, Nancy, France) for the poplar leaf content analyses, Aude Fauvet for the analysis of total phenolics, and Didier Le Thiec for the lyophilization of the samples. We also thank Irène Hummel for advices in statistical analyses, Rantanplan for inspiration, Josselin Montarry, Elisabeth Fournier, Stéphane De Mita, Claire Fourrey, and Katherine Hayden for valuable comments on earlier drafts of this manuscript.

\section{SUPPLEMENTARY MATERIAL}

The Supplementary Material for this article can be found online at: https://www.frontiersin.org/articles/10.3389/fpls.2018.01396/ full\#supplementary-material

Duplessis, S., Cuomo, C. A., Lin, Y. C., Aerts, A., Tisserant, E., VeneaultFourrey, C., et al. (2011). Obligate biotrophy features unraveled by the genomic analysis of rust fungi. Proc. Natl. Acad. Sci. U.S.A. 108, 9166-9171. doi: 10.1073/ pnas. 1019315108

Duplessis, S., Spanu, P. D., and Schirawski, J. (2013). "Biotrophic Fungi (Powdery Mildews, Rusts, and Smuts)," in The Ecological Genomics of Fungi, ed. F. Martin (Hoboken, NJ: John Wiley \& Sons, Inc.), 149-168. doi: 10.1002/97811187358 93.ch7

Farber, D. H., and Mundt, C. C. (2017). Effect of plant age and leaf position on susceptibility to wheat stripe rust. Phytopathology 107, 412-417. doi: 10.1094/ PHYTO-07-16-0284-R

Ficke, A., Gadoury, D. M., and Seem, R. C. (2002). Ontogenic resistance and plant disease management: a case study of grape powdery mildew. Phytopathology 92, 671-675. doi: 10.1094/PHYTO.2002.92. 6.671

Gamm, M., Héloir, M. C., Bligny, R., Vaillant-Gaveau, N., Trouvelot, S., Alcaraz, G., et al. (2011). Changes in carbohydrate metabolism in Plasmopara viticola-infected grapevine leaves. Mol. Plant Microbe. Interact. 24, 1061-1073. doi: 10.1094/MPMI-02-11-0040

Gavrilets, S., and Scheiner, S. M. (1993). The genetics of phenotypic plasticity. V. Evolution of reaction norm shape. J. Evol. Biol. 6, 31-48. doi: 10.1046/j.14209101.1993.6010031.x

Gilchrist, M. A., Sulsky, D. L., and Pringle, A. (2006). Identifying fitness and optimal life-history strategies for an asexual filamentous fungus. Evolution 60, 970-979. doi: 10.1554/05-261.1

Giorcelli, A., Vietto, L., Anselmi, N., and Gennaro, M. (1996). Influence of clonal susceptibility, leaf age and inoculum density on infections by Melampsora laricipopulina races E1 and E3. Eur. J. Forest Pathol. 26, 323-331. doi: 10.1111/j.14390329.1996.tb01078.x

Guinet, C., Boutigny, A. L., Vialle, A., Hamelin, R. C., Frey, P., and Ioos, R. (2016). Simultaneous monitoring and quantification of Melampsora alliipopulina and Melampsora larici-populina on infected poplar leaves using a duplex real-time PCR assay. Plant Pathol. 65, 380-391. doi: 10.1111/ppa. 12426 
Hakulinen, J., Sorjonen, S., and Julkunen-Tiitto, R. (1999). Leaf phenolics of three willow clones differing in resistance to Melampsora rust infection. Physiol. Plant. 105, 662-669. doi: 10.1034/j.1399-3054.1999.105410.x

Jarosz, A. M., and Davelos, A. L. (1995). Effects of disease in wild plant populations and the evolution of pathogen aggressiveness. New Phytolo. 129, 371-387. doi: 10.1111/j.1469-8137.1995.tb04308.x

Jensen, B., and Munk, L. (1997). Nitrogen-induced changes in colony density and spore production of Erysiphe graminis f.sp. hordei on seedlings of six spring barley cultivars. Plant Pathol. 46, 191-202. doi: 10.1046/j.1365-3059.1997.d01-224.x

Johnson, J. D., and Kim, Y. (2005). The role of leaf chemistry in Melampsora medusae infection of hybrid poplar: effects of leaf development and fungicide treatment. Can. J. Forest Res. 35, 763-771. doi: 10.1139/x05-009

Johnston, S. F., Cohen, M. F., Torok, T., Meentemeyer, R. K., and Rank, N. E. (2016). Host phenology and leaf effects on susceptibility of California Bay Laurel to Phytophthora ramorum. Phytopathology 106, 47-55. doi: 10.1094/ PHYTO-01-15-0016-R

Kardin, M. K., and Groth, J. V. (1989). Density-dependent fitness interactions in the bean rust fungus. Phytopathology 79, 409-412. doi: 10.1094/Phyto-79-409

Katsuya, K., and Green, G. J. (1967). Reproductive potentials of races $15 \mathrm{~b}$ and 56 of wheat stem rust. Can. J. Bot. 45, 1077-1091. doi: 10.1139/b67-113

Knott, E. A., and Mundt, C. C. (1991). Latent period and infection efficiency of Puccinia recondita f. sp. tritici populations isolated from different wheat cultivars. Phytopathology 81, 435-439. doi: 10.1094/Phyto-81-435

Kolmer, J. A., and Leonard, K. J. (1986). Genetic selection and adaptation of Cochliobolus heterostrophus to corn hosts with partial resistance. Phytopathology 76, 774-777. doi: 10.1094/Phyto-76-774

Lannou, C. (2012). Variation and selection of quantitative traits in plant pathogens. Annu. Rev. Phytopathol. 50, 319-338. doi: 10.1146/annurev-phyto-081211173031

Larson, P. R., and Isebrands, J. G. (1971). The plastochron index as applied to developmental studies of cottonwood. Can. J. Forest Res. 1, 1-11. doi: 10.1139/ x71-001

Leonard, K. J. (1969). Selection in heterogeneous populations of Puccinia graminis f. sp. avenae. Phytopathology 59, 1851-1857.

Madan, M., and Thind, K. S. (1998). Physiology of Fungi. New Delhi: APH Publishing Corporation.

Merry, A. M., Evans, K. J., Corkrey, R., and Wilson, S. J. (2013). Coincidence of maximum severity of powdery mildew on grape leaves and the carbohydrate sink-to-source transition. Plant Pathol. 62, 842-850. doi: 10.1111/j.1365-3059. 2012.02691.x

Pap, P., Rankovic, B., and Masirevic, S. (2013). Effect of temperature, relative humidity and light on conidial germination of oak powdery mildew (Microsphaera alphitoides Griff. et Maubl.) under controlled conditions. Arch. Biol. Sci. 65, 1069-1077. doi: 10.2298/ABS1303069P

Pariaud, B., Ravigné, V., Halkett, F., Goyeau, H., Carlier, J., and Lannou, C. (2009a). Aggressiveness and its role in the adaptation of plant pathogens. Plant Pathol. 58, 409-424. doi: 10.1111/j.1365-3059.2009.02039.x

Pariaud, B., Robert, C., Goyeau, H., and Lannou, C. (2009b). Aggressiveness components and adaptation to a host cultivar in wheat leaf rust. Phytopathology 99, 869-878. doi: 10.1094/PHYTO-99-7-0869

Pei, M. H., Ruiz, C., Harris, J., and Hunter, T. (2003). Quantitative inoculations of poplars with Melampsora larici-populina. Eur. J. Plant Pathol. 109, 269-276. doi: 10.1023/A:1022822503139

Pei, M. H., Ruiz, C., Hunter, T., Arnold, G. M., and Bayon, C. (2002). Quantitative relationships between inoculum of Melampsora larici-epitea and corresponding disease on Salix. Plant Pathol. 51, 443-453. doi: 10.1046/j.1365-3059.2002. 00739.x

Philibert, A., Desprez-Loustau, M. L., Fabre, B., Frey, P., Halkett, F., Husson, C., et al. (2011). Predicting invasion success of forest pathogenic fungi from species traits. J. Appl. Ecol. 48, 1381-1390. doi: 10.1111/j.1365-2664.2011.02039.x
Robert, C., Bancal, M. O., and Lannou, C. (2002). Wheat leaf rust uredospore production and carbon and nitrogen export in relation to lesion size and density. Phytopathology 92, 762-768. doi: 10.1094/PHYTO.2002.92.7.762

Robert, C., Bancal, M. O., and Lannou, C. (2004). Wheat leaf rust uredospore production on adult plants: influence of leaf nitrogen content and Septoria tritici blotch. Phytopathology 94, 712-721. doi: 10.1094/PHYTO.2004.94.7.712

Rotem, J., Cohen, Y., and Bashi, E. (1978). Host and environmental influences on sporulation in vivo. Annu. Rev. Phytopathol. 16, 83-101. doi: 10.1146/annurev. py.16.090178.000503

Royer, M., Larbat, R., Le Bot, J., Adamowicz, S., and Robin, C. (2013). Is the $\mathrm{C}: \mathrm{N}$ ratio a reliable indicator of $\mathrm{C}$ allocation to primary and defence-related metabolisms in tomato? Phytochemistry 88, 25-33. doi: 10.1016/j.phytochem. 2012.12.003

Scheiner, S. M. (1993). Genetics and evolution of phenotypic plasticity. Annu. Rev. Ecol. Syst. 24, 35-68. doi: 10.1146/annurev.es.24.110193.000343

Shain, L., and Miller, J. B. (1982). Pinocembrin: an antifungal compound secreted by leaf glands of eastern cottonwood [Populus deltoides]. Phytopathology 72, 877-880 doi: 10.1094/Phyto-72-877

Sharma, J. K., Heather, W. A., and Winer, P. (1980). Effect of leaf maturity and shoot age of clones of Populus species on susceptibility to Melampsora larici-populina. Phytopathology 70, 548-554. doi: 10.1094/Phyto-70-548

Trapero-Casas, A., and Kaiser, W. J. (1992). Influence of temperature, wetness period, plant age, and inoculum concentration on infection and development of Ascochyta blight of chickpea. Phytopathology 82, 589-596. doi: 10.1094/Phyto82-589

Turechek, W. W., Mahaffee, W. F., and Ocamb, C. M. (2001). Development of management strategies for hop powdery mildew in the Pacific Northwest. Plant Health Prog. 2:8. doi: 10.1094/PHP-2001-0313-01-RS

Turechek, W. W., and Stevenson, K. L. (1998). Effects of host resistance, temperature, leaf wetness, and leaf age on infection and lesion development of pecan scab. Phytopathology 88, 1294-1301. doi: 10.1094/PHYTO.1998.88.12. 1294

Ullah, C., Unsicker, S. B., Fellenberg, C., Constabel, C. P., Schmidt, A., Gershenzon, J., et al. (2017). Flavan-3-ols are an effective chemical defense against rust infection. Plant Physiol. 175, 1560-1578. doi: 10.1104/pp.17.00842

Vagiri, M., Johansson, E., and Rumpunen, K. (2017). Phenolic compounds in black currant leaves - an interaction between the plant and foliar diseases? J. Plant Interact. 12, 193-199. doi: 10.1080/17429145.2017.1316524

Valkama, E., Koricheva, J., Salminen, J. -P., Helander, M., Saloniemi, I., Saikkonen, K., et al. (2005). Leaf surface traits: overlooked determinants of birch resistance to herbivores and foliar micro-fungi? Trees 19, 191-197. doi: 10.1007/s00468-004-0380-5

Xavier, A. A., da Silva, A. C., da Silva Guimarães, L. M., Matsuoka, K., Hodges, C. S., and Alfenas, A. C. (2015). Infection process of Puccinia psidii in Eucalyptus grandis leaves of different ages. Trop. Plant Pathol. 40, 318-325. doi: 10.1007/ s40858-015-0043-7

Yegorov, I., Grognard, F., Mailleret, L., and Halkett, F. (2017). Optimal resource allocation for biotrophic plant pathogens. IFAC PapersOnline 50, 3154-3159. doi: 10.1016/j.ifacol.2017.08.328

Conflict of Interest Statement: The authors declare that the research was conducted in the absence of any commercial or financial relationships that could be construed as a potential conflict of interest.

Copyright (C) 2018 Maupetit, Larbat, Pernaci, Andrieux, Guinet, Boutigny, Fabre, Frey and Halkett. This is an open-access article distributed under the terms of the Creative Commons Attribution License (CC BY). The use, distribution or reproduction in other forums is permitted, provided the original author(s) and the copyright owner(s) are credited and that the original publication in this journal is cited, in accordance with accepted academic practice. No use, distribution or reproduction is permitted which does not comply with these terms. 